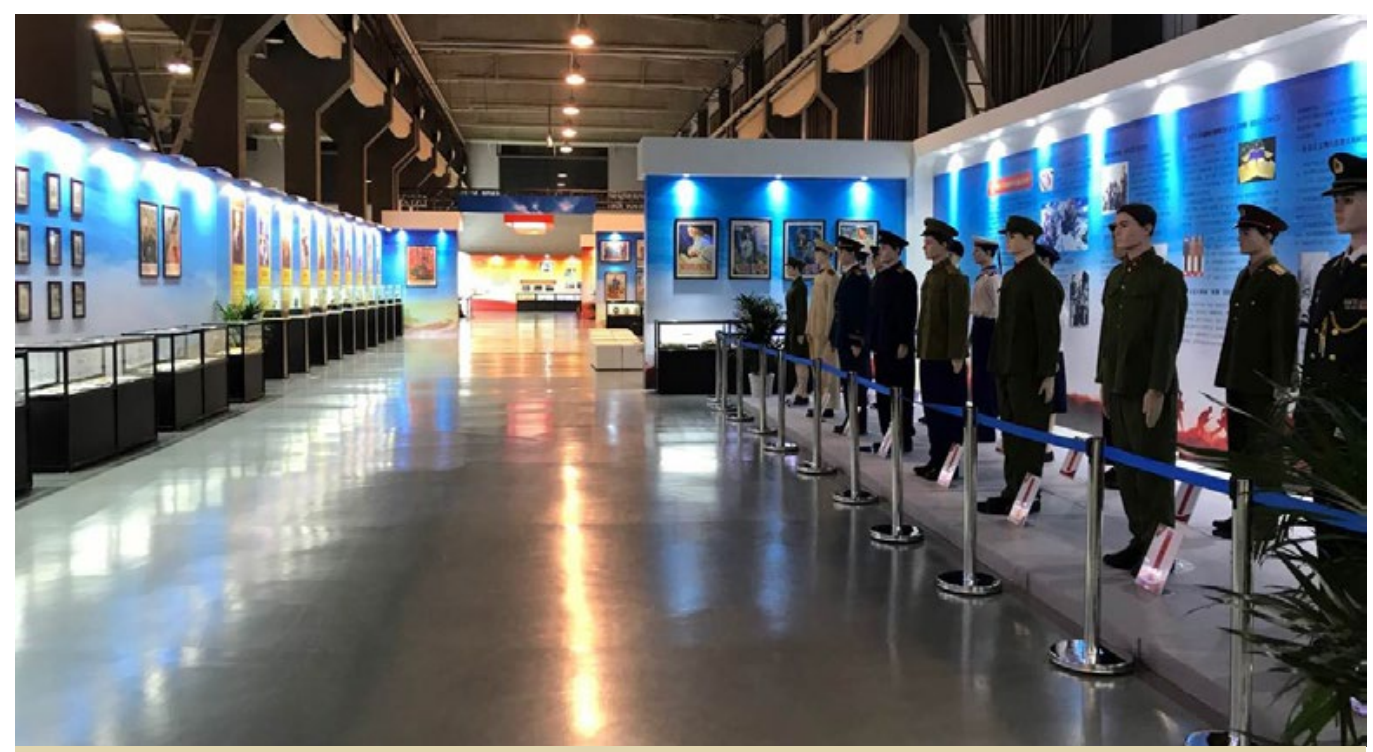

Figure 1: View of the exhibition in Chaoyang Park Beijing. August 2017. Author's photo.

\title{
Collecting the Red Era in Contemporary China
}

\section{Emily Williams}

Since the 1980s the Chinese Communist Party has condemned the Cultural Revolution as 'ten years of chaos'. Nevertheless, so far there has been very little discussion on the topic in the public sphere in China. This essay looks into how private collections of red relics can be used to confront this void in China's recent past. It argues that collected objects play a much more complex role in history production than we may think, as they contribute to the construction of narratives, put forth counternarratives, and fragment the very idea of historical narrative altogether.
The summer of 2017 saw the ninetieth anniversary of the founding of China's People's Liberation Army. The occasion was marked not only by a massive rally that was streamed live on television-an unequivocal display of military power overseen by President Xi Jinping (Phillips 2017)-but also by a number of exhibitions. This fact, in and of itself, is unsurprising, given the boom in museums and so-called 'red tourism' in China in recent years. One such exhibition was held at the Urban Planning Museum in Beijing's Chaoyang Park, and was significant, less for its content and curation than for its organisers: it was a collaborative effort between the government of Chaoyang 
district in Beijing, the well-known flea market of Panjiayuan, and the Red Collectors Committee of the China National Collectors Association.

The Red Collectors Association is a nationally approved body that brings together collectors of Maoist material culture. For this exhibition, they provided over 1,500 so-called 'red relics' (hongse wenwu), drawn from the private collections of association members from around the country. Somewhat peculiarly, the exhibition almost entirely excluded discussion of the objects themselves, using them as simple accessories for the broader narrative that was expounded in great detail on the walls of the exhibition. While it could be argued that in doing so the curators missed an opportunity to add an extra layer of interest to their exhibition, such a strategy is perhaps not surprising given the sensitivity that surrounds the writing of modern Chinese history. Indeed, while the curators worked hard to portray the red relics as an intrinsic part of China's 'red legacy', in reality these relics occupy a somewhat contradictory place in contemporary China.
'Red relics' are objects from the 'Red Era', a term that can indicate any period after the founding of the Chinese Communist Party (CCP) in 1921, but most often refers to the decades between 1949 and 1976. Many collections are strongest in objects from the Cultural Revolution (1966-1976), a highly controversial period that saw the production of abundant and hugely diverse material culture. Since the 1980s, the Cultural Revolution has been condemned by the CCP as 'ten years of chaos', but it continues to be the subject of both celebratory and condemnatory memories in China. These contradictory remembrances are well known, even if there is little discussion on the topic in the public sphere. For example, the Cultural Revolution was not mentioned at the Chaoyang Exhibition, except for brief mention of the country's foreign policy at that time. If we want, therefore, to understand how collections can be used to confront China's recent past, we need to look beyond public exhibitions, and consider the practices of China's red collectors.
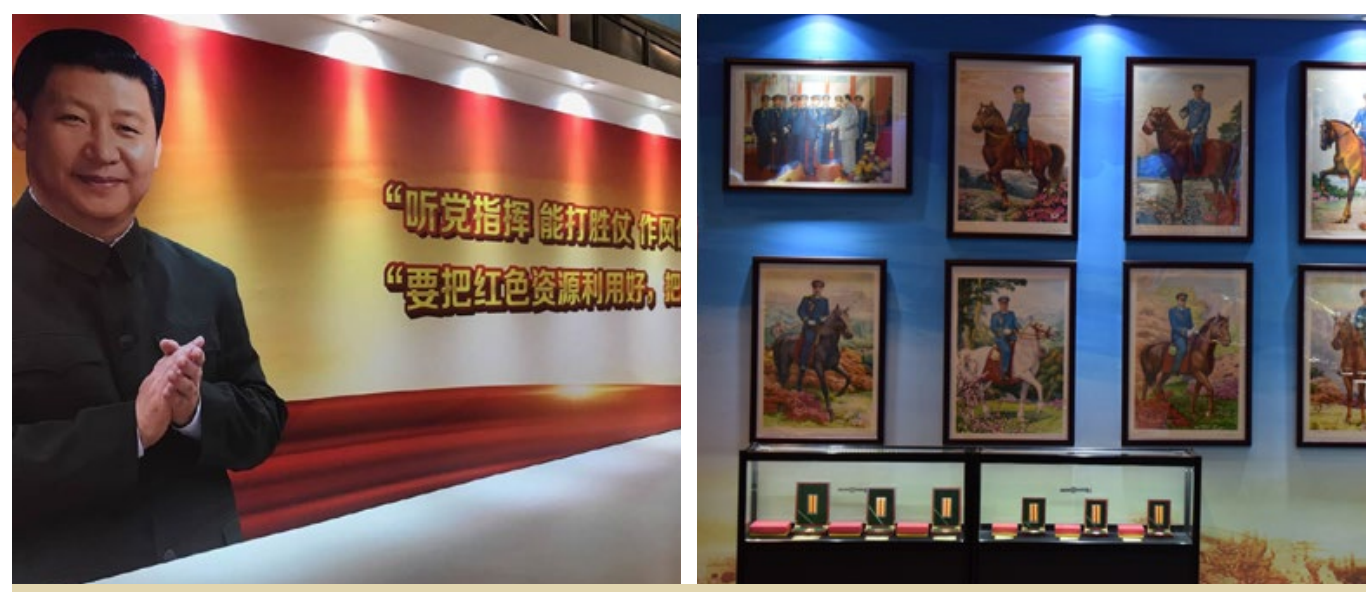

(left) Figure 2: A photograph of Chinese President Xi Jinping in the entrance hall of the exhibition. The second quote reads: 'Utilise red resources, develop the red tradition, and pass on the red gene well.' The exhibition tried to position the red relics on display as part of the 'red resources' Xi mentions. (right) Figure 3: Posters depicting some of the Ten Marshals and, below, a selection of military medals. Photos taken by the author. 


\section{Fragmented Narratives: Li Jun}

Collected objects play a much more complex role in constructing historical narratives than we may think. We might be tempted to think of a collection as a systematic accumulation of historical traces. By acquiring and then protecting these remains, the collector ensures the survival of these remnants of history, and thus ensures historical continuity. However, theorists, such as Susan Stewart (1992), problematise the relationship between history and a collection, and argue that the collected object, rather than preserving history, in fact destroys it. Indeed, the implicit search for an internal relationship between the present and the past that is embedded in the act of collecting is marked by an absolute disruption, whereby entering a collection, the object must be removed from the context from which it previously derived value and meaning. The relationship, then, is not rooted in historical reality, but rather represents an aesthetic denial of historical value. For this reason, Stewart calls a collection the 'total aestheticisation of use value' (1992, 151), and the place where history is transformed into space: a transformation that is marked most profoundly by the establishment of boundaries. According to Jean Baudrillard, a collection is defined by the establishment of a series-sets into which objects can be classified, defined by the collector, and with reference to the collector (1996, 96-97). In other words, in the classic manifestation of a collection, classification does not put forward an historical narrative, but rather fragments any such narrative through serialisation.

Cultural Revolution objects, despite their origin in perhaps the most anticapitalist period in modern Chinese history, lend themselves remarkably easily to the commodification that serialisation necessitates. For instance, Li Jun, a collector from Ningbo, Zhejiang province, began collecting Mao badges five years ago, primarily as a hobby and as a way to relieve the stress of his high-level job in a fashion company. According to an interview that I had with him in June 2016, since then he has acquired over thirty thousand Mao badges, as well as military and labour medals. He stores them in immaculately organised stacks of shelves in his spacious modern flat, organised primarily by aesthetic similarity. There are trays of young Mao on his way to Anyuan, Mao in wheat fields, Mao in every imaginable pose and in front of every imaginable background, all neatly arranged.
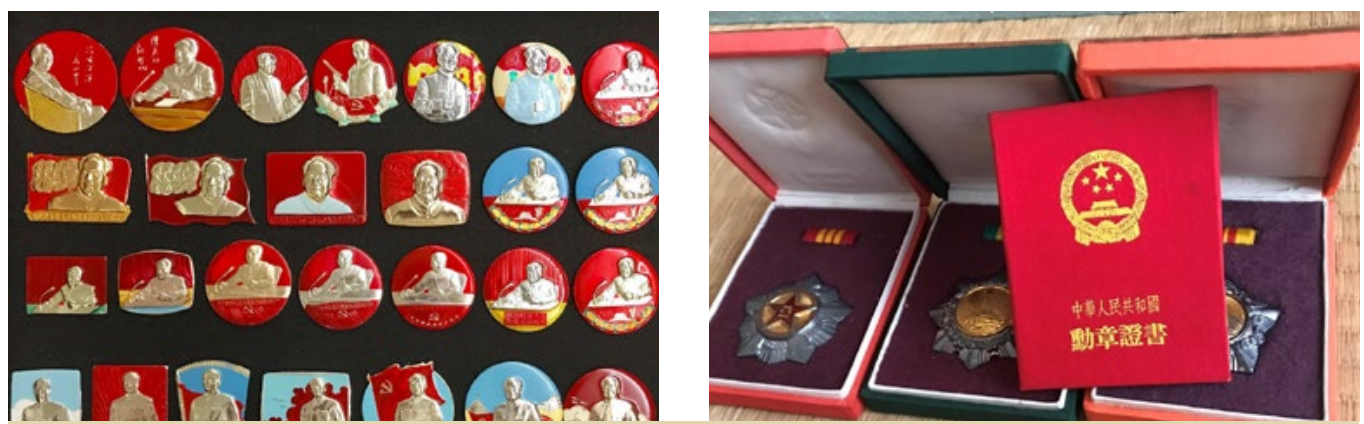

(Left) Figure 4: A selection of Li Jun's Mao badges. (Right) Figure 5: 1950s military medals and the accompanying 'meritorious medal certificate', which contains details of their original owners. Images supplied by Li Jun, 2017 

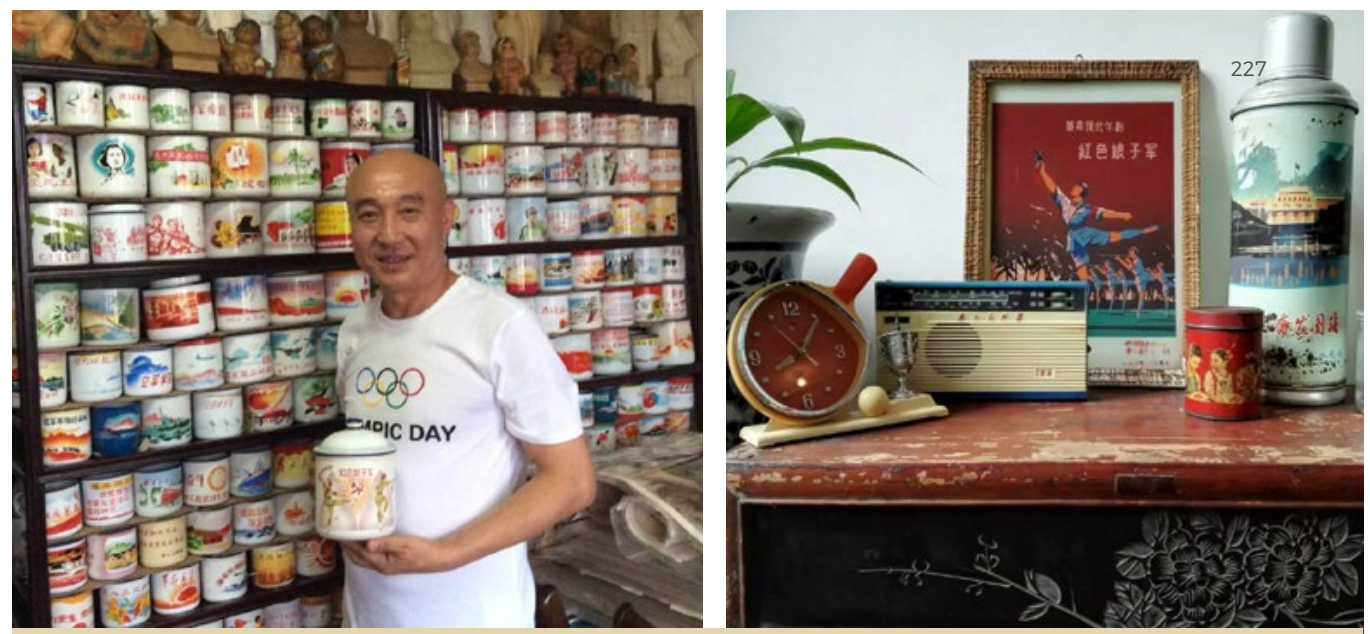

(Left) Figure 6: Beijing collector Dong Zhongchao in front of his shelf of enamel mugs, which are organised thematically. (Right) Figure 7: While Dong started off collecting posters, he now collects anything that reflects the red aesthetic of the Mao years. Image supplied by Dong Zhongchao, 2016.

Leading figures in the Collectors Association encourage members to do research on and through their objects. $\mathrm{Li}$ argues that the work units (danwei) and military units listed on the back of many badges can be a starting point for understanding cultural production during the Cultural Revolution. But, in fact, organising badges by appearance does not encourage their insertion into a historical narrative, but rather into an aesthetic series in which history is fragmented and objects gain their importance only from their appearance and their similarity (or dissimilarity) to their neighbours.

Badges have been a popular collectible in China since the early 1990s, and to be considered a collector of any merit, one must have some ten thousand unique objects. But the real way to distinguish oneself in this highly competitive field is to complement bulk objects with more rare examples. In Li's case, the pride of his collection is a small number of model worker medals and military medals, primarily from the 1950s. These are marked out by Li's attempt to historicise them: he not only collects the medals, but also tries to acquire the personal documents of each medal's former owner. In doing so, he is able to attach a personal narrative to the objects, but the process also highlights the historical distance between their original context and the present.

\section{Image-Driven Archiving: Dong Zhongchao}

Other collectors deal with the problem of historicisation in different ways. Beijing collector Dong Zhongchao-whom I interviewed in 2016 and 2017-began trading and then collecting Mao-era posters in the 1990 s, but in the 2000s he started to expand the scope of his collection. It now includes all sorts of daily life objects, such as mugs, plates, basins, teapots and thermoses, textiles and clothing, mirrors, toys, and other items that similarly share the Maoist aesthetic of the time. Alongside this, he also collects things such as Mao busts and other sculptures, editions of the Little Red Book, and Mao badges, but Dong makes no discursive or practical distinction between 

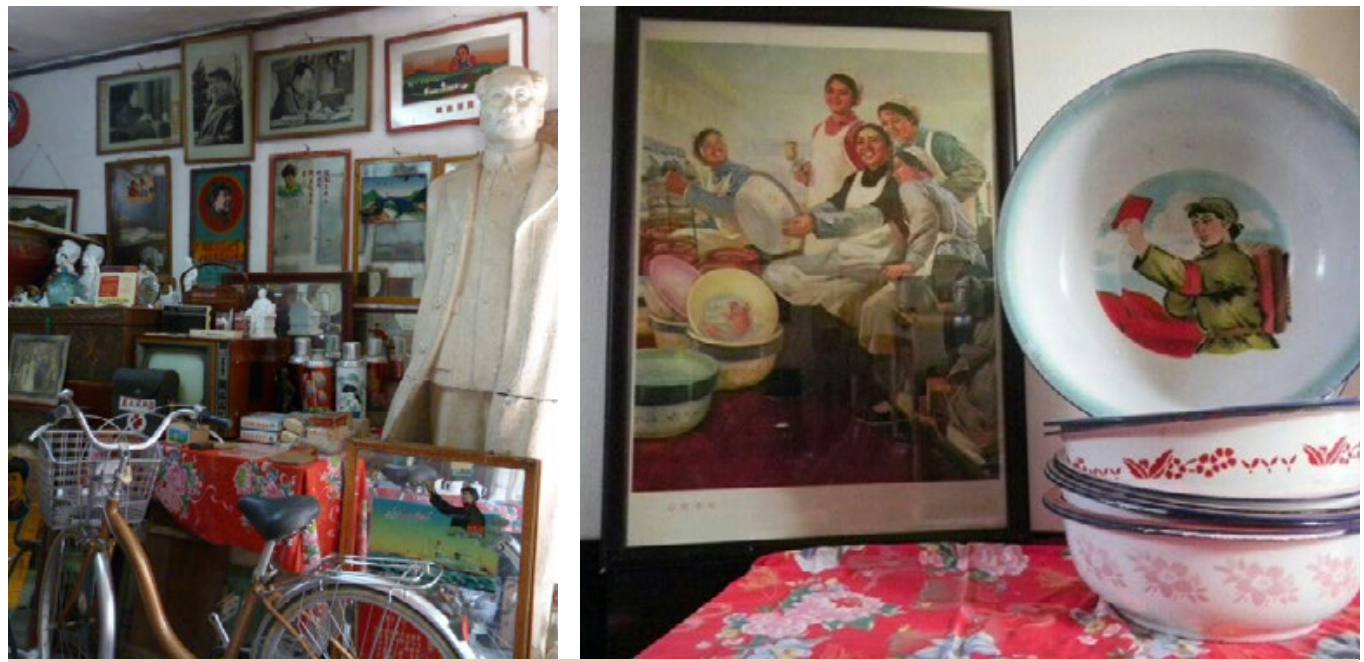

(Left) Figure 8: Dong's storeroom, containing statues, mirrors, a television, a bicycle, and myriad other objects. (Right) Figure 9: Dong's archival strategy: matching imagery from posters with objects from daily life. Photo provided by Dong Zhongchao, 2016.

these and those daily life objects, mixing them all together both on the shelves and in his conversations.

Compared to Li's ordered trays, Dong's memorabilia are stacked and heaped in an overflowing storeroom, as well as scattered throughout his home. But his collection is also meticulously digitised, and it is in his digital collection that his conception of history becomes apparent. In addition to organising his objects thematically, by place, or by period as is common in many collections, Dong takes particular pleasure in matching up his items with correspondent visual depictions in propaganda posters (see images 9 and 10).

It could be suggested that this type of archiving practice seeks to legitimise the imagery presented in the posters by demonstrating that the materiality of the life depicted in the posters can be recollected. Possessing the physical objects visible in posters seems to endorse the historicity of the poster image, suggesting the reality of the poster messages by virtue of the reality of the objects depicted in it.
But the strategy also works the other way around, as an attempt to heal the fissure in the relationship between past and present by using the poster imagery to provide the missing context for the collected objects. This strategy has a number of implications for the conceptualisation of the relationship between collected object and historical narrative.

Dipesh Chakrabarty has argued that modern historical consciousness is formed by taking objects that are contemporaneous with us and seeing them as 'relics' from the past (2008, 238-239). Doing this limits the historical object's power because it denies the possibility of a lived relation with the present-any impact the object still has is just an effect of the past. Thus, the historical object is doubly denied agency: firstly, by its removal from the context in which it originally had meaning; and secondly, by its reconceptualisation as a 'relic'. Dong's approach attempts to restore an agency to the object: combining the actual objects with their visual portrayal in posters is an attempt to construct a new, more active 

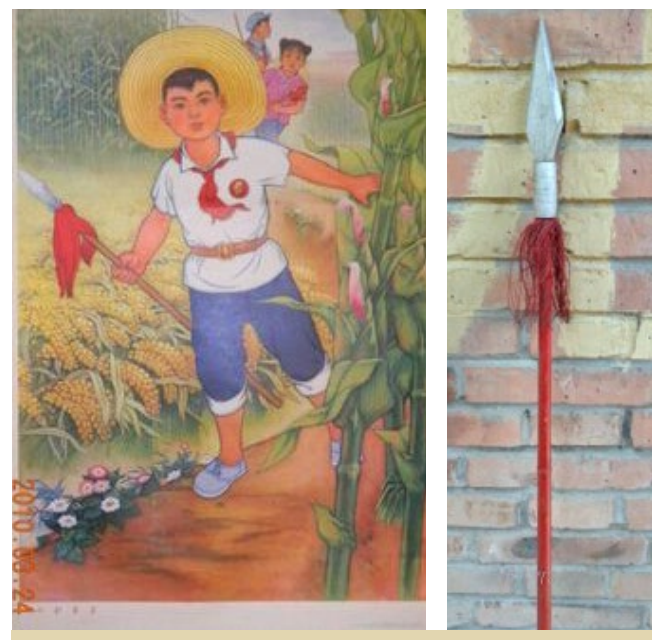

Figure 10: Dong's efforts to match poster imagery with objects goes beyond just objects of 'daily life'. Photo provided by Dong Zhongchao, 2016. way of engaging with both posters and real objects, transporting the viewer back to the Mao era, and reaffirming the validity of the values of that time, a period that he sees as characterised by greater equality compared to the corruption and individualism of contemporary Chinese society.

\section{Total Recall?}

Susan Stewart argues that the problem with trying to use relics to overcome the gap between past and present is that objects recall not the experience lived, but the experience voluntarily remembered (1992, 145). However, in today's China, where public discussion of memories of the Cultural Revolution remains limited, it is precisely memories, including those expressed through collecting practices, which are needed. While the Chaoyang Exhibition presented a clear narrative of the military's smooth rise to power and prominence, other collecting practices hold the possibility of telling more complicated stories. That these stories lack coherence is not a bad thing. Indeed, what is needed are multiple, overlapping, even conflicting narratives. If we understand the archival practices of red collectors as exercises in historical memory and as forms of historical writing, we can appreciate them for their diversity. Objects and their collections construct narratives, put forth counter-narratives, and fragment the very idea of narratives altogether. When considered collectively, they represent one of the ways in which contemporary China is attempting to deal with its difficult recent past. 
This text is taken from Gilded Age: A Year of Chinese Labour, Civil Society, and Rights, Made in China Yearbook 2017, edited by Ivan Franceschini and Nicholas Loubere, published 2018 by ANU Press, The Australian National University, Canberra, Australia.

$$
\text { doi.org/10.22459/MIC.04.2018.42 }
$$

